\title{
Targeting molecular tumor types
}

In this issue, the Focus on Pan-Cancer Analysis examines the similarities and differences among the genomic and cellular alterations found in the first dozen tumor types to be profiled by The Cancer Genome Atlas (TCGA) Research Network. This first look across cancers offers new tools in genomics and bioinformatics and the prospect of repurposing targeted therapies to be directed by the molecular pathology of tumors in addition to their clinical classification.

$(5$ uccesses in cancer therapy have stemmed from the 'divide and conquer' approach. Cytogenetic identification of $B C R-A B L$ translocation led to improvements in the treatment of chronic myelogenous leukemia. Recognition of subtypes of breast cancer by molecular profiling has enhanced the matching of therapy to disease with some improvement in outcomes. Non-small-cell lung cancer can be divided into nearly a hundred molecular types, some of which present mutated targets-such as EGFR and $A L K$ - for directed therapeutics ( $A m$. Soc. Clin. Oncol. Educ. Book 2013, 127-134, 2013).

At the same time, genomic approaches have identified hundreds of recurrently mutated genes influencing cancer cell survival that fall into a small number of molecular pathways or networks (Science 339,1546-1558, 2013). To delimit these altered pathways, TCGA has catalogued aberrations in the DNA, chromatin and RNA of genomes from thousands of tumors relative to matched normal cellular genomes and has analyzed the epigenetic, protein and cell signaling consequences of these aberrations.

In this issue (p. 1111) and in the associated Focus online (http://www.nature.com/ng/focus/tcga), the Pan-Cancer Analysis Working Group presents the tools, resources and analytical strategies they have developed to identify shared and tissue-specific mechanisms operating across the cancers in the TCGA-12 data release. With the release of the accompanying data sets, it has finally become possible to reanalyze oncogenic perturbations within each tumor type using the multiple controls offered by other tumor types. When such analysis is carried out, commonalities at the molecular level begin to emerge among tumors of disparate tissues of origin.
Consortia characterizing cancer genomes (TCGA, Pediatric Cancer Genome Consortium and International Cancer Genome Consortium) have achieved a remarkable thing in that they have created a collaborative framework in which the key genes and pathways that contribute to cancer can be rapidly and securely identified. Such a framework will allow these groups to quickly reapply existing methods to new questions as they arise. The framework comes with a commitment to the rapid distribution of data to the research community, meaning that the usefulness of findings can be assessed independently in the prevention, early diagnosis, prognosis and therapeutic targeting of cancers.

Consortium productivity presents a publishing challenge. Each individual research article must meet consortium standards as well as the criteria of anonymous peer review. Then, the articles need to be coordinated among collaborating and competing journals, and, after publication, the whole compendium needs to be readily comprehensible to advance the needs of the cancer research community. Thanks to our sponsor, Illumina, and the TCGA authors, we have been able to meet this challenge, providing the research articles in a fully open format and also connecting the main analytical themes using the hypertext thread concept pioneered by the Encyclopedia of DNA Elements (ENCODE) project (http://www.nature.com/encode/threads). We have reworked the papers to discuss mutational drivers, network models, mutational signatures from the environment and pathogens, and data discovery and visualization. As this Focus marks the beginning of pan-cancer analysis, future directions for this research are discussed both in the threads and in our podcast http://www.nature.com/multimedia/podcast/ng/ ng-2013-10-17.mp3. 